\title{
Satisfaction with Medications Prescribed for Osteoarthritis: A Cross-Sectional Survey of Patients and Their Physicians in the United States
}

\author{
Rebecca L. Robinson (D) - Thomas J. Schnitzer • Sophie Barlow • \\ Mia Berry · Andrew G. Bushmakin · Joseph C. Cappelleri • \\ Leslie Tive · Jessica Jackson · James Jackson · Lars Viktrup
}

Received: October 28, 2021 / Accepted: December 17, 2021 / Published online: January 13, 2022

(C) The Author(s) 2022

\begin{abstract}
Introduction: Satisfaction with medications prescribed for osteoarthritis (OA) varies; this study aimed to determine the factors associated with satisfaction in US patients and their physicians.

Methods: This point-in-time study used the Adelphi OA Disease Specific Programme (physicians identified from public lists reported on nine consecutive patients diagnosed with OA [any joint]: physicians and patients completed questionnaires). Patient's demographic, clinical, and treatment characteristics associated with patient-reported and physician-rated overall satisfaction with, and expectations of
\end{abstract}

Supplementary Information The online version contains supplementary material available at https:// doi.org/10.1007/s40122-021-00350-0.

R. L. Robinson $(\varangle) \cdot$ L. Viktrup

Lilly Research Labs, Eli Lilly and Company, Lilly

Corporate Center, Indianapolis, IN 46285, USA

e-mail: rlrobinson@lilly.com

\section{T. J. Schnitzer}

Northwestern University Feinberg School of

Medicine, Chicago, IL, USA

S. Barlow · M. Berry · J. Jackson · J. Jackson Adelphi Real World, Bollington, Cheshire, UK

A. G. Bushmakin · J. C. Cappelleri

Pfizer Inc, Groton, CT, USA

L. Tive

Pfizer Inc, New York, NY, USA effectiveness of, medication for OA were assessed using multivariate linear regression.

Results: Responses from 572 patients (mean age 64.9 years, $60.5 \%$ female) currently prescribed medication for $\mathrm{OA}$ and 153 physicians (81 primary care, 35 rheumatologists, 37 orthopedic surgeons) were analyzed. Pain intensity was moderate or severe for $59.4 \%$ of patients. Greater patient-reported overall satisfaction with medication was significantly associated with (standardized beta, 95\% confidence interval) exercise $(0.12,0.03-0.20)$, comorbid other musculoskeletal or painful conditions (vs none) $(0.15,0.06-0.24)$, and physicians' report that the best control had been achieved $(0.12$, 0.03-0.20); lack of efficacy was among factors associated with worse satisfaction. Greater patient-reported expectation of effectiveness was significantly associated with exercise $(0.12$, 0.03-0.21) and the most troublesome joint not being a knee, hip, or their back $(0.08$, 0.01-0.14). Greater physician-rated overall satisfaction with medication was significantly associated with their report that the best control had been achieved $(0.18,0.11-0.26)$, the most troublesome joint being a knee (0.08, 0.01-0.14), comorbid other musculoskeletal or painful conditions $(0.07,0.01-0.12)$, obesity (0.06, 0.00-0.11), and female patients (0.06, $0.00-0.11)$; lack of efficacy and adverse events/tolerability issues were among factors associated with worse satisfaction. For physicians, their report that the best control had 
been achieved $(0.19,0.11-0.27)$, the most troublesome joint being a knee $(0.08$, 0.00-0.15), improving (vs stable) OA $(0.15$, $0.07-0.24)$, and uncertain duration of OA (0.11, $0.02-0.21$ ) were associated with greater perception that the medication was meeting patients' efficacy expectations.

Conclusion: Although efficacy was strongly associated with both patients' and physicians' satisfaction with medication, other factors were also important, including exercise (for patients), tolerability (for physicians), and knee OA (for physicians).

Keywords: Analgesics; Prescription analgesic medication; Real-world clinical practice; Patient satisfaction; Treatment satisfaction

\section{Key Summary Points}

Why carry out this study?

Satisfaction with medications prescribed for osteoarthritis (OA) varies, and the factors associated with satisfaction need further clarification

The factors associated with satisfaction have not been reported from the perspectives of both patients and their physicians in US clinical practice settings

This study investigated which patient demographic, clinical, and treatment characteristics were associated with satisfaction with, and expectations of the effectiveness of, medications currently prescribed for $\mathrm{OA}$

\section{What was learned from the study?}

Among the factors associated with greater overall satisfaction with, and expectation of effectiveness of, medications for OA, exercise was important for patients and tolerability and the most troublesome joint being a knee were important for physicians; for both patients and physicians, lack of efficacy was most strongly associated with worse overall satisfaction
Understanding the factors that are associated with patient satisfaction, and how these differ from those associated with physician satisfaction, could aid shared decision-making, optimize treatment, and improve satisfaction for patients with $\mathrm{OA}$

\section{INTRODUCTION}

Pain management is a priority for patients with osteoarthritis (OA) [1] and a multidisciplinary treatment approach is often recommended, including education, exercise, and pharmacological treatment where indicated [2-4]. The pharmacological options include analgesics (such as acetaminophen, oral and topical nonsteroidal anti-inflammatory drugs [NSAIDs], duloxetine, and opioids including tramadol) and intra-articular therapies (corticosteroids, and viscosupplements such as hyaluronic acid), but they can be ineffective or unsuitable for some patients [2-4]. Satisfaction with treatment for OA varies [5-7].

Patient satisfaction with pain management has been the focus of quality metric initiatives [8]. Although treatment satisfaction is generally considered to be related to adherence, quality of life, and clinical outcomes [9], findings have been inconsistent for patients with chronic pain [10-13]. Generally, treatment satisfaction has been linked to prior expectations $[9,14,15]$ and may be associated with specific treatment attributes $[9,15]$.

There are limited data relating to the determinants of satisfaction in patients with OA. Factors predicting satisfaction with surgery for OA have been investigated [16], but the factors associated with satisfaction with pharmaceutical treatments for OA have been less well studied. Satisfaction with OA medications has been associated with changes in symptoms $[5,17,18]$ but other factors that may be implicated, such as demographic, clinical, and treatment characteristics, require further clarification. In addition, drivers of treatment satisfaction from 
the patient's perspective may differ from drivers of treatment satisfaction from the physician's perspective. Understanding the factors that are associated with patient satisfaction, and any differences from those associated with physician satisfaction, could help optimize treatment and improve satisfaction for patients with OA. Shared decision-making is likely to be improved when physicians are aware of what is important to patients, and how this might differ from their own perspective.

The aim of this study was to determine the factors associated with satisfaction with medications prescribed for $\mathrm{OA}$, from the perspectives of both patients and their physicians in the USA.

\section{MATERIALS AND METHODS}

Data were collected in the USA between February and May 2017 using the Adelphi OA Disease Specific Programme ${ }^{\mathrm{TM}}$ (DSP), point-in-time, real-world methodology published previously [19] (Supplementary Material, Fig. S1). The Adelphi DSPs are large, multinational studies of clinical practice designed to capture a cross section of robust, real-world data reflecting current demographics, clinical presentation, and treatment patterns for a range of diseases [19]. The Adelphi DSP methodology was granted exemption from requiring ethics approval by the Western Institutional Review Board as it was considered to pose minimal risk to patients and physicians. All patients provided consent.

\section{Study Population}

Participating physicians (primary care physicians, rheumatologists, and orthopedic surgeons) were identified from public lists, and those making treatment decisions for at least 10 patients with OA in a typical month completed an online survey about their practice patterns and online patient record forms on their next nine consecutive adult patients diagnosed with and consulting for OA. The diagnostic criteria for OA, and other disease characteristics (such as joints affected or time since diagnosis), were not stipulated. Each of the patients was invited to voluntarily self-complete a paper-based questionnaire about their OA.

\section{Measures}

\section{Demographics, Clinical Characteristics, and Treatment Characteristics}

Patients reported their education level and whether a caregiver was responsible for their daily needs. Patients scored their pain intensity ("How would you rate the intensity of your pain, on average, over the last week?", 11-point numeric rating scale from $0=$ no pain to $10=$ worst possible pain), assessed their functional limitations due to OA (Western Ontario and McMaster Universities Osteoarthritis Index [WOMAC] $^{1}$ [20] Physical Function subscale, numeric rating scale ranging from 0 to 10 , with higher scores indicating worse function), and completed the EQ-5D-5L to assess general health status across five dimensions (mobility, self-care, usual activities, pain/discomfort, and anxiety/depression) [21]. Patients also reported the type of healthcare provider from whom they had ever received OA care (primary care, rheumatologist, orthopedic surgeon, pain specialist/anesthesiologist, other), current use of nonpharmacologic therapies (exercise [nonweight bearing, weight bearing, "exercise", yoga/pilates], avoidance of painful activities, self-aids [such as ice or heat therapy, insoles/cushioning in shoes, dietary supplements, home remedies], or other services [such as acupuncture, therapist/psychotherapist, physical therapy]). Patients reported use (current, prior, never) of over-the-counter (OTC) medications ("Have you ever taken any medication that you have bought over the counter from a pharmacy or supermarket specifically to help treat your osteoarthritis symptoms?"). They also reported whether they have difficulties with adhering to medications and out-ofpocket expenses associated with their treatment.

Physicians recorded the patient's age, gender, ethnicity, employment, and insurance status. Physicians reported general clinical

\footnotetext{
1 (C) 1996 Nicholas Bellamy. WOMAC® is a registered
} trademark of Nicholas Bellamy (CDN, EU, USA). 
characteristics including the patient's smoking status, disability status, presence of obesity (body mass index $>30 \mathrm{~kg} / \mathrm{m}^{2}$ ), presence of comorbidities (cardiologic, endocrine, neurologic/psychologic, respiratory, chronic low back pain, other musculoskeletal or painful conditions [osteoporosis, neuropathic pain, rheumatoid arthritis, connective tissue disease, migraine], cancer, obesity, other, none), the medications associated with their comorbidities, and their assessment of the patient's risk of cardiovascular or gastrointestinal issues. Physicians reported clinical characteristics specific to OA including the time since the patient's diagnosis, details of affected joints (number, location, "most troublesome" joint), rate of OA progression (improving, stable, deteriorating slowly, deteriorating rapidly), and risk of falls, and whether OA pain was "controlled" ("Is this the best control that can realistically be achieved for this patient?") or considered treatment resistant ("Do you believe this patient's osteoarthritis pain is treatment resistant?"). Physicians also reported current prescription medications for OA pain (NSAIDs, opioids [including tramadol, nontramadol opioids, and combination drugs with opioids], corticosteroids [intra-articular or oral], viscosupplements, other treatments [such as acetaminophen, capsaicin, glycosaminoglycans]), number of OA treatment regimens (ever received, at class level), and treatment issues (lack of efficacy, patient decision, drug interactions/comorbidities, adverse events/tolerability, worries about addiction, cost, or access issues).

\section{Patient-Reported Satisfaction with, and Expectations of Effectiveness of, Medication for $\mathrm{OA}$}

Overall satisfaction with medications prescribed for OA was assessed using a 5-point Likert scale. Patients were asked "Which of the following options best describes your overall satisfaction with the prescribed medicine(s) for your osteoarthritis?" (options: very satisfied, 1; somewhat satisfied, 2; neither satisfied nor dissatisfied, 3; somewhat dissatisfied, 4; very dissatisfied, 5).

Expectations of the effectiveness of medications prescribed for $\mathrm{OA}$ were assessed using a 5-point Likert scale. Patients were asked "How is your current medicine(s) meeting your level of expectation in relation to how effective it is for your OA?" (options: it is a great deal more effective than I expected, 1 ; it is more effective than I expected, 2; it matches my expectations, 3 ; it is less effective than I expected, 4 ; it is much less effective than I expected, 5).

Patients were asked to rate their satisfaction with different attributes of medications on a 5 -point Likert scale from 1 to 5 (extremely dissatisfied, 1; somewhat dissatisfied, 2; neither dissatisfied nor satisfied, 3; somewhat satisfied, 4; extremely satisfied, 5), in response to "How satisfied are you with your prescribed medicine(s) in relation to...". Eleven treatment attributes were appended to the question and scores combined to reflect four categories: pain relief (short-term relief, long-term relief, ease pain quickly), functional change (helps keep you mobile/active, allows return to usual activities, helps maintain independence), tolerability (side effects of medication), and convenience of medications (clear/simple instructions, convenient to take/fit into schedule, easy to remember to take, cost of medicine).

\section{Physician-Rated Satisfaction with, and Expectations of Effectiveness of, Medication for $\mathrm{OA}$}

Overall satisfaction with medications prescribed for OA was assessed using a 5-point Likert scale. Physicians were asked "Which of the following statements best describes your satisfaction with the patient's prescribed therapy (to treat their OA pain)?" (options: very satisfied, 1 ; somewhat satisfied, 2; neither satisfied nor dissatisfied, 3; somewhat dissatisfied, 4 ; very dissatisfied, 5 ).

Expectations of the effectiveness of medications prescribed for OA were assessed using a 5-point Likert scale. Physicians were asked "How do you believe the current drug therapy regimen meets the patient's own expectations in relation to efficacy achieved?" (options: greatly exceeds expectations, 1 ; exceeds expectations, 2; matches expectations, 3; less than expected, 4; much less than expected, 5). 


\section{Statistical Analysis}

To enable investigation of treatment satisfaction, patients eligible for the analyses were those participants in the Adelphi OA DSP who were currently prescribed oral medication(s) for OA (more than 30 days) or intra-articular medications for $\mathrm{OA}$ and who had answered the question on overall satisfaction with current treatment. An oversample per physician of one patient who had tried at least three medications for their OA pain was included. Patients were excluded if the only joint affected was the back as this may be characteristic of a separate clinical entity rather than OA.

Pain and functional limitation scores were classified as mild (score $0-3$ ), moderate (4-6), or severe (7-10). Comorbid chronic low back pain had a high prevalence, so this was analyzed as a separate comorbidity category; owing to the low prevalence of other musculoskeletal and painful conditions, these were combined for analyses.

Patient demographic, clinical, and treatment characteristics were assessed for association with patient-reported and physician-rated overall satisfaction with, and expectations of the effectiveness of, medications for OA. For all outcomes, identification of relevant factors was based on bivariate comparisons according to patient-reported overall satisfaction (dichotomized as satisfied [very satisfied/somewhat satisfied] or neutral/dissatisfied [neither satisfied nor dissatisfied/somewhat dissatisfied/ very dissatisfied]), using Student's $t$ test (continuous variables) or Pearson's chi-square (categorical variables) [22]. In the contingency table analysis with an expected cell count of less than 5, Fisher's exact test (for 2-by-2 tables) [22] or Fisher's generalized exact test was used (for $r$ by-c tables, where $r$ or $c$ or both exceed 2) [23].

Multiple linear regressions were performed for all satisfaction and expectations outcomes, the covariates including race and gender as well as all those with two-tailed $p<0.25$ in the bivariate comparisons $[24,25]$. The final set of factors were identified using the LASSO (least absolute shrinkage and selection operator) approach [26] with cross validation. LASSO identifies covariates with non-zero coefficients (which have an impact on outcome) by introducing a penalty parameter that forces, or shrinks, select coefficients to zero, which then excludes them from the prediction process. The factors with non-zero coefficients were included in the final models, with $p<0.05$ considered significant. Standardized beta coefficients were used to evaluate the relative importance of each independent factor's association with outcome [27]: the higher the absolute value of the beta coefficient, the stronger its relative effect on outcome (i.e., a beta of -0.9 has a stronger effect than a beta of +0.8 ). For ease of interpretation, outcomes were transformed as necessary so that a positive beta coefficient was associated with greater satisfaction/expectations and a negative beta coefficient was associated with worse satisfaction/expectations. Normality was tested in the regression models by plotting the residuals against the fitted values and examining a QQ plot to observe any deviations.

Concordance between patient and physician outcomes was assessed using a (quadratic weighted) kappa statistic [28].

Data were managed and analyzed using SPSS version 7.5 (SPSS Inc., Chicago, IL, USA) and Stata version 16.1 (StataCorp, College Station, TX, USA).

\section{RESULTS}

The physician cohort comprised 153 physicians (81 in primary care, 35 rheumatologists, 37 orthopedic surgeons). A total of 964 patients with OA completed the Adelphi OA DSP questionnaire. Of these, 572 patients were eligible for the current analyses: patients were excluded for not currently taking medication $(n=238)$, for having solely OA of the back $(n=51)$, and for failing to answer the question on overall satisfaction with current treatment $(n=103)$.

Among the 572 patients, mean age was 64.9 years, $60.5 \%$ were female, $76.9 \%$ were white, and the most troublesome joint was a knee for $49.7 \%$ (Table 1). Pain intensity was moderate or severe for $59.4 \%$ of patients, and functional limitations due to OA were moderate or severe for $41.8 \%$ of patients (Table 1 ). Comorbidities included chronic low back pain 
Table 1 Demographic and clinical characteristics of patients currently prescribed medication for $\mathrm{OA}$

\begin{tabular}{|c|c|}
\hline & $\begin{array}{l}\text { Patients } \\
(n=572)\end{array}$ \\
\hline Age, mean $(\mathrm{SD})$ & $64.9(11.3)$ \\
\hline Female, $n(\%)$ & $346(60.5)$ \\
\hline White/Caucasian, $n(\%)$ & $440(76.9)$ \\
\hline Unemployed, $n(\%)^{\mathrm{a}, \mathrm{b}}$ & $321(56.4)$ \\
\hline Body mass index $>30 \mathrm{~kg} / \mathrm{m}^{2}, n(\%)$ & $224(39.2)$ \\
\hline Time since diagnosis (weeks), mean $(\mathrm{SD})^{\mathrm{c}}$ & $135.7(172.2)$ \\
\hline Number of affected joints, mean $(S D)^{\mathrm{d}}$ & $3.6(2.7)$ \\
\hline \multicolumn{2}{|l|}{ Most troublesome joint, $n(\%)$} \\
\hline Knee & $284(49.7)$ \\
\hline Hip & $120(21.0)$ \\
\hline Back & $99(17.3)$ \\
\hline Other & $155(27.1)$ \\
\hline \multicolumn{2}{|c|}{ Pain intensity, on average, over the last week, $n(\%)^{\mathrm{e}}$} \\
\hline Mild (0-3) & $230(40.6)$ \\
\hline Moderate (4-6) & $225(39.8)$ \\
\hline Severe $(7-10)$ & $111(19.6)$ \\
\hline \multicolumn{2}{|l|}{ Functional limitations due to OA, $n(\%)^{\mathrm{f}}$} \\
\hline Mild (0-3) & $309(58.2)$ \\
\hline Moderate (4-6) & $141(26.6)$ \\
\hline Severe $(7-10)$ & $81(15.3)$ \\
\hline \multicolumn{2}{|c|}{ Healthcare provider (providing OA care, ever), $n$ (\%) } \\
\hline Rheumatologist & $209(36.5)$ \\
\hline Primary care physician & $441(77.1)$ \\
\hline Orthopedic surgeon & $230(40.2)$ \\
\hline Pain specialist/anesthesiologist & $58(10.1)$ \\
\hline Other & $174(30.4)$ \\
\hline \multicolumn{2}{|l|}{ Comorbidities, $n(\%)^{\mathrm{g}}$} \\
\hline Chronic low back pain & $89(15.6)$ \\
\hline $\begin{array}{l}\text { Other musculoskeletal or painful } \\
\text { conditions }\end{array}$ & $100(17.5)$ \\
\hline Osteoporosis & $51(8.9)$ \\
\hline
\end{tabular}

Table 1 continued

\begin{tabular}{|c|c|}
\hline & $\begin{array}{l}\text { Patients } \\
(n=572)\end{array}$ \\
\hline Neuropathic pain & $24(4.2)$ \\
\hline Rheumatoid arthritis & $16(2.8)$ \\
\hline Connective tissue disease & $16(2.8)$ \\
\hline Migraine & $12(2.1)$ \\
\hline $\begin{array}{l}O A \text { osteoarthritis, } S D \text { standard deviation } \\
{ }^{\text {a } D u e ~ t o ~ O A ~}(n=22) \text { or not due to OA }(n= \\
\text { bSample size } n=569 \\
{ }^{\mathrm{c}} \text { Sample size } n=302 \\
{ }^{\mathrm{d}} \text { Number of affected joints: } 1(n=119), 2(n= \\
\text { more }(n=302) \\
{ }^{\mathrm{e}} \text { Sample size } n=566 \\
{ }^{\mathrm{f}} \text { Sample size } n=531 \\
{ }^{\mathrm{g}} \text { For other comorbidities, see Supplementary } \\
\text { Table S2 }\end{array}$ & y Material, \\
\hline
\end{tabular}

(15.6\%) and other musculoskeletal or painful conditions (17.5\%) (Table 1). Medications currently prescribed for OA for more than 30 days included NSAIDs for $77.9 \%$, opioids (including tramadol) for $30.5 \%$, corticosteroids for $12.4 \%$, viscosupplementation for $4.8 \%$, and other treatments for $28.5 \%$ of patients. Further patient demographics, and clinical and treatment characteristics, are provided in Supplementary Material (Tables S1-S3). The 153 physicians were predominantly male $(72.5 \%)$ and had been in practice for at least 15 years (77.8\%); further physician characteristics are provided in Supplementary Material (Table S4).

A total of $67.8 \%$ of 572 patients were very satisfied or somewhat satisfied with their current medication for $\mathrm{OA}$, and physicians were satisfied with the treatment of $74.3 \%$ of their patients. Conversely, physicians were satisfied with the treatment of $54.4 \%$ of the 103 patients excluded from analyses for failing to answer the question on overall satisfaction with current treatment $(p=0.0007$ for satisfaction rates for the patients included in the analyses $[n=572]$ vs those excluded $[n=103]$ ).

Bivariate analyses identified multiple factors of interest (Supplementary Material, Tables S1-S3), the final set of factors relevant for 
Table 2 Factors associated with level of patient-reported overall satisfaction with currently prescribed medication for OA

\begin{tabular}{llc}
\hline Factor & $\begin{array}{l}\text { Standardized } \\
\text { beta }\end{array}$ & 95\% CI \\
\hline Treatment issue: lack of efficacy & -0.26 & $-0.37,-0.14$ \\
OA progression: deteriorating rapidly (reference: stable) & -0.16 & $-0.25,-0.06$ \\
Comorbid: other musculoskeletal or painful conditions (reference: none) & 0.15 & $0.06,0.24$ \\
Pain: moderate (4-6) (reference: mild 0-3) & -0.12 & $-0.22,-0.03$ \\
Physician-reported best control achieved & 0.12 & $0.03,0.20$ \\
Exercise & 0.12 & $0.03,0.20$ \\
OA progression: deteriorating slowly (reference: stable) & -0.11 & $-0.21,-0.01$ \\
Treatment issue: drug interactions/comorbidities & -0.08 & $-0.14,-0.01$
\end{tabular}

$C I$ confidence interval, $O A$ osteoarthritis

Multivariate linear regression. Constant: coefficient 0.00 (95\% CI $-0.09,0.09)$. Number of observations $=403 . F(34$, $101)=20.72$. Prob $>F=0.00 . R^{2}=0.47$. Root mean square error $=0.76$. Positive beta value is associated with better satisfaction and negative value associated with worse satisfaction; the higher the absolute value of the beta coefficient, the stronger the effect. All factors in the final model that were associated with outcome at $p<0.05$ are shown

Table 3 Factors associated with level of patient-reported expectation of effectiveness of currently prescribed medication for $\mathrm{OA}$

\begin{tabular}{lrr}
\hline Factor & Standardized beta & 95\% CI \\
\hline Treatment issue: lack of efficacy & -0.15 & $-0.28,-0.02$ \\
OA progression: deteriorating slowly (reference: stable) & -0.14 & $-0.24,-0.04$ \\
Currently buy OTC medication (reference: never bought OTC) & -0.14 & $-0.26,-0.03$ \\
Used to buy OTC medication (reference: never bought OTC) & -0.12 & $-0.24,-0.00$ \\
Exercise & 0.12 & $0.03,0.21$ \\
Time since diagnosis: $>$ 3 years (reference: $1-3$ years) & -0.11 & $-0.22,-0.00$ \\
Most troublesome joint: hip & -0.10 & $-0.19,-0.00$ \\
Most troublesome joint: other & 0.08 & $0.01,0.14$ \\
\hline
\end{tabular}

$C I$ confidence interval, $O A$ osteoarthritis, $O T C$ over-the-counter

Multivariate linear regression. Constant: coefficient 0.00 (95\% CI $-0.09,0.09)$. Number of observations $=398 . F(42$, $95)=12.43$. Prob $>F=0.00 . R^{2}=0.45$. Root mean square error $=0.79$. Positive beta value is associated with better expectation and negative value associated with worse expectation; the higher the absolute value of the beta coefficient, the stronger the effect. All factors in the final model that were associated with outcome at $p<0.05$ are shown

inclusion in the models being determined by LASSO (Supplementary Material, Fig. S2). Multivariate analysis confirmed which demographic, clinical, and treatment characteristics were significantly associated with patient- reported overall satisfaction with medications for OA (Table 2), patient-reported expectation of effectiveness of medications for OA (Table 3), and patient-reported satisfaction with attributes of medications for OA (Table 4). Multivariate 
Table 4 Factors significantly associated with level of patient-reported satisfaction with attributes of currently prescribed medication for OA

\begin{tabular}{|c|c|c|c|}
\hline $\begin{array}{l}\text { Attribute of } \\
\text { medication }\end{array}$ & Factor associated with level of satisfaction with attribute & $\begin{array}{l}\text { Standardized } \\
\text { beta }\end{array}$ & $95 \% \mathrm{CI}$ \\
\hline \multirow[t]{5}{*}{ Pain relief ${ }^{a}$} & Treatment issue: lack of efficacy & -0.16 & $\begin{array}{r}-0.28 \\
-0.05\end{array}$ \\
\hline & EQ-5D-5L & 0.15 & $0.01,0.30$ \\
\hline & $\begin{array}{l}\text { Comorbid: other musculoskeletal or painful conditions } \\
\text { (reference: none) }\end{array}$ & 0.10 & $0.01,0.18$ \\
\hline & Physician-reported best control achieved & 0.09 & $0.01,0.17$ \\
\hline & Exercise & 0.09 & $0.01,0.17$ \\
\hline \multirow[t]{9}{*}{ Functional change $^{\mathrm{b}}$} & EQ-5D-5L & 0.28 & $0.14,0.43$ \\
\hline & Time since diagnosis: don't know (reference: $1-3$ years) & 0.15 & $0.04,0.25$ \\
\hline & Treatment issue: lack of efficacy & -0.14 & $\begin{aligned}- & 0.24 \\
- & 0.04\end{aligned}$ \\
\hline & Pain: moderate (4-6) (reference: mild 0-3) & -0.13 & $\begin{array}{r}-0.21 \\
-0.04\end{array}$ \\
\hline & Current opioid use & -0.12 & $\begin{aligned}- & 0.21 \\
- & 0.02\end{aligned}$ \\
\hline & Healthcare provider ever: orthopedic surgeon & 0.12 & $0.04,0.20$ \\
\hline & $\begin{array}{l}\text { Comorbid: other musculoskeletal or painful conditions } \\
\text { (reference: none) }\end{array}$ & 0.10 & $0.02,0.18$ \\
\hline & Time since diagnosis: $<1$ year (reference: & 0.09 & $0.00,0.17$ \\
\hline & $1-3$ years $)$ & & \\
\hline \multirow[t]{3}{*}{ Tolerability $^{c}$} & Treatment issue: adverse events or tolerability & -0.23 & $\begin{array}{r}-0.36 \\
-0.09\end{array}$ \\
\hline & Functional limitation: severe $(7-10)$ (reference: mild $0-3$ ) & -0.17 & $\begin{array}{l}-0.30 \\
-0.04\end{array}$ \\
\hline & Pain: moderate (4-6) (reference: mild 0-3) & -0.15 & $\begin{array}{r}-0.27 \\
-0.04\end{array}$ \\
\hline
\end{tabular}


Table 4 continued

\begin{tabular}{llcc}
\hline $\begin{array}{l}\text { Attribute of } \\
\text { medication }\end{array}$ & Factor associated with level of satisfaction with attribute & $\begin{array}{l}\text { Standardized } \\
\text { beta }\end{array}$ & $\mathbf{9 5 \%}$ CI \\
\hline Convenience $^{\mathrm{d}}$ & Lines of OA medication: $\geq 3$ (reference: 1$)$ & -0.16 & -0.30, \\
& & -0.03 \\
Ethnicity: Black, Asian, and minority ethnic & -0.16 & -0.25, \\
& & -0.06 \\
Number of medications for concomitant conditions: $>4$ & 0.16 & $0.05,0.28$ \\
$\quad$ (reference: 0-2) & -0.13 & -0.22, \\
Most troublesome joint: knee & -0.04 \\
& & -0.25, \\
Pain: moderate (4-6) (reference: mild 0-3) & -0.01 \\
& & -0.25, \\
Treatment-resistant OA pain & -0.13 & -0.01 \\
& & $0.03,0.21$ \\
Age: $<55$ years (reference: 66-75 years) & 0.12 & $0.01,0.19$ \\
Nonpharmacologic therapy: other & 0.10 & $0.01,0.17$ \\
Number of medications for concomitant conditions: 3-4 & 0.09 & \\
(reference: 0-2) & & \\
\hline
\end{tabular}

$C I$ confidence interval, $O A$ osteoarthritis

Multivariate linear regression. Positive beta value is associated with better satisfaction and negative value associated with worse satisfaction; the higher the absolute value of the beta coefficient, the stronger the effect. All factors in the final model that were associated with outcome at $p<0.05$ are shown

${ }^{\mathrm{a}}$ Constant: coefficient 0.00 (95\% CI $\left.-0.10,0.10\right)$. Number of observations $=430 . F(25,102)=10.22$. Prob $>F=0.00$. $R^{2}=0.33$. Root mean square error $=0.85$

${ }^{\mathrm{b}}$ Constant: coefficient $0.00(95 \% \mathrm{CI}-0.09,0.09)$. Number of observations $=408 . F(34,100)=16.94$. Prob $>F=0.00$. $R^{2}=0.45$. Root mean square error $=0.78$

'Constant: coefficient 0.00 (95\% CI $-0.11,0.11)$. Number of observations $=478 . F(17,104)=5.50$. Prob $>F=0.00$. $R^{2}=0.14$. Root mean square error $=0.94$

${ }^{\mathrm{d}}$ Constant: coefficient $0.00(95 \% \mathrm{CI}-0.11,0.11)$. Number of observations $=365 . F(29,94)=11.19$. Prob $>F=0.00$. $R^{2}=0.24$. Root mean square error $=0.91$

analysis confirmed which patient demographic, clinical, and treatment characteristics were significantly associated with physician-rated overall satisfaction with medications for OA (Table 5) and physicians' views of how OA medications had met patient expectations of efficacy (Table 6). No abnormalities were found in the regression models when checking for normality (data not shown).

\section{Patient-Reported Satisfaction with, and Expectations of Effectiveness of, Medication for OA}

Patient ratings for overall satisfaction are shown in Fig. 1a. Greater patient-reported overall satisfaction was significantly associated with exercise, the presence of comorbid other musculoskeletal or painful conditions (compared with none), and physicians' report that the best control had been achieved (Table 2). 
Table 5 Factors associated with level of physician-rated overall satisfaction with currently prescribed medication for OA

\begin{tabular}{lcc}
\hline Factor & Standardized beta & 95\% CI \\
\hline Treatment issue: lack of efficacy & -0.30 & $-0.39,-0.20$ \\
Treatment-resistant OA pain & -0.18 & $-0.29,-0.06$ \\
Best control achieved & 0.18 & $0.11,0.26$ \\
OA progression: deteriorating slowly (reference: stable) & -0.17 & $-0.24,-0.10$ \\
OA progression: deteriorating rapidly (reference: stable) & -0.15 & $-0.22,-0.08$ \\
Treatment issue: adverse events or tolerability & -0.15 & $-0.22,-0.07$ \\
Pain: severe (7-10) (reference: mild 0-3) & -0.09 & $-0.17,-0.01$ \\
Unemployment not due to OA (reference: employed) & -0.08 & $-0.13,-0.02$ \\
Most troublesome joint: knee & 0.08 & $0.01,0.14$ \\
Lines of OA medication: 2 (reference: 1) & -0.07 & $-0.13,-0.01$ \\
Comorbid: other musculoskeletal or painful conditions (reference: none) & 0.07 & $0.01,0.12$ \\
Obesity & 0.06 & $0.00,0.11$ \\
Female & 0.06 & $0.00,0.11$ \\
\hline
\end{tabular}

$C I$ confidence interval, $O A$ osteoarthritis

Multivariate linear regression. Constant: coefficient 0.00 (95\% CI $-0.07,0.07)$. Number of observations $=565 . F(28$, $112)=23.79$. Prob $>F=0.00 . R^{2}=0.56$. Root mean square error $=0.68$. Positive beta value is associated with better satisfaction and negative value associated with worse satisfaction; the higher the absolute value of the beta coefficient, the stronger the effect. All factors in the final model that were associated with outcome at $p<0.05$ are shown

Lack of efficacy had the strongest effect associated with worse overall satisfaction (Table 2). Other factors significantly associated with worse patient-reported overall satisfaction were moderate pain intensity (compared with mild pain), OA that was deteriorating (compared with stable $\mathrm{OA}$ ), and drug interactions/comorbidities (Table 2).

Patient ratings for expectations of effectiveness are shown in Fig. 1b. Greater patient-reported expectation of effectiveness was significantly associated with exercise and the most troublesome joint being other than a knee, hip, or back (i.e., hands/fingers, neck, shoulder, wrist, ankle, foot, elbow). Lack of efficacy had the strongest effect associated with worse expectation of effectiveness (Table 3). Other factors significantly associated with worse expectation of effectiveness were OA that was slowly deteriorating (compared with stable OA), use of OTC medications, diagnosis more than
3 years ago, and the most troublesome joint being a hip (Table 3 ).

Patient ratings for satisfaction with the attributes of medication are shown in Fig. 2. When considering medication pain relief, EQ$5 \mathrm{D}-5 \mathrm{~L}$, exercise, and the presence of comorbid other musculoskeletal or painful conditions (compared with none) were among the factors significantly associated with greater patient-reported satisfaction (Table 4). With respect to functional change afforded by medication, EQ$5 \mathrm{D}-5 \mathrm{~L}$, care by an orthopedic surgeon (ever), and the presence of comorbid other musculoskeletal or painful conditions (compared with none) were among the factors associated with greater patient-reported satisfaction, and opioids were associated with worse satisfaction (Table 4). When considering medication tolerability, severe functional limitation (compared with mild) was among the factors associated with worse satisfaction (Table 4). With respect 
Table 6 Factors associated with physician rating of how the currently prescribed medication for OA is meeting the patient's expectation of efficacy

\begin{tabular}{lcl}
\hline Factor & Standardized beta & 95\% CI \\
\hline Treatment issue: lack of efficacy & -0.21 & $-0.30,-0.12$ \\
Best control achieved & 0.19 & $0.11,0.27$ \\
OA progression: improving (reference: stable) & 0.15 & $0.07,0.24$ \\
OA progression: deteriorating rapidly (reference: stable) & -0.12 & $-0.19,-0.06$ \\
Treatment issue: adverse events or tolerability & -0.12 & $-0.22,-0.03$ \\
Time since diagnosis: don't know (reference: 1-3 years) & 0.11 & $0.02,0.21$ \\
Pain: moderate (4-6) (reference: mild 0-3) & -0.10 & $-0.18,-0.02$ \\
Most troublesome joint: knee & 0.08 & $0.00,0.15$ \\
\hline
\end{tabular}

$C I$ confidence interval, $O A$ osteoarthritis

Multivariate linear regression. Constant: coefficient 0.00 (95\% CI $-0.09,0.09)$. Number of observations $=568 . F(16$, $112)=14.86$. Prob $>F=0.00 . R^{2}=0.39$. Root mean square error $=0.79$. Positive beta value is associated with better expectation and negative value associated with worse expectation; the higher the absolute value of the beta coefficient, the stronger the effect. All factors in the final model that were associated with outcome at $p<0.05$ are shown

to the convenience of medications, taking multiple medications for comorbidities and younger age (less than 55 years relative to those 66-75 years of age) were among the factors associated with better satisfaction and ethnicity (black, Asian, and minority ethnic groups compared with Caucasian) was associated with worse satisfaction (Table 4).

\section{Physician-Rated Satisfaction with, and Expectations of Effectiveness of, Medication for $\mathrm{OA}$}

Physician ratings for overall satisfaction are shown in Fig. 3a. Greater physician-rated overall satisfaction was significantly associated with their report that the best control had been achieved, the most troublesome joint being a knee, the presence of comorbid other musculoskeletal or painful conditions (compared with none), obesity, and female patients (Table 5). Lack of efficacy had the strongest effect associated with worse overall satisfaction (Table 5). Other factors significantly associated with worse overall satisfaction were adverse events/tolerability issues, pain that was considered to be treatment resistant, OA that was deteriorating (compared with stable OA), severe pain intensity (compared with mild pain), unemployment that was not due to OA, and taking two lines of OA medication (compared with one line) (Table 5).

Physicians' views of how medications were meeting patients' efficacy expectations are shown in Fig. 3b. Physicians' report that the best control had been achieved, the most troublesome joint being a knee, improving OA (compared with stable OA), and uncertain duration of $\mathrm{OA}$ were significantly associated with greater expectations (Table 6). Lack of efficacy had the strongest effect associated with worse expectations (Table 6). Also significantly associated with worse expectations were adverse events/tolerability issues, OA that was rapidly deteriorating (compared with stable OA), and moderate pain intensity (compared with mild pain) (Table 6).

\section{Concordance Between Patients and Physicians}

There was moderate agreement between patient and physician ratings of overall satisfaction with $55.2 \%$ of 572 ratings matched (Cohen's weighted kappa $=0.57 ; p<0.0001)$. There was moderate agreement between patient and 

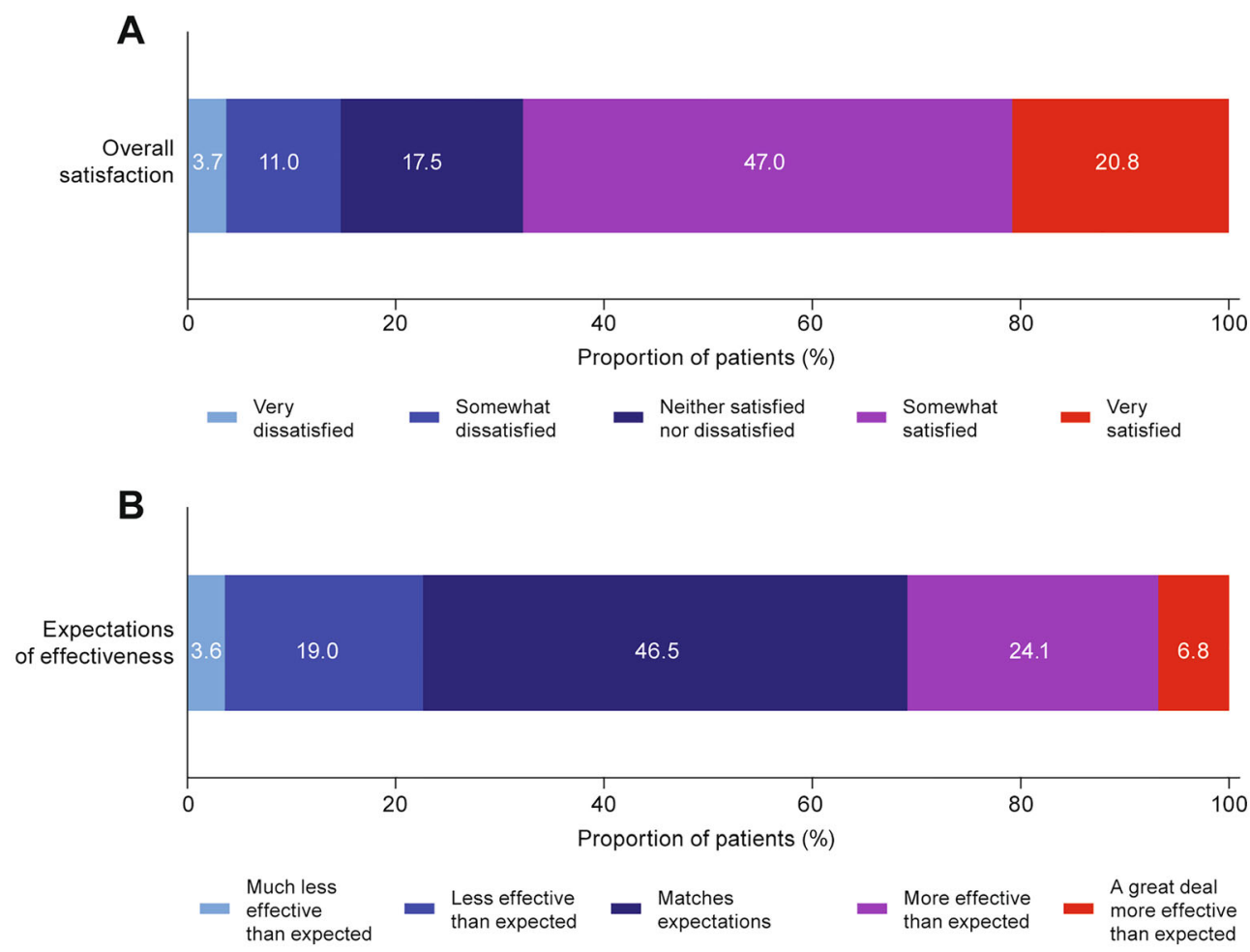

Fig. 1 Patient-reported a overall satisfaction with ${ }^{\mathrm{a}}$, and b expectations of effectiveness of , currently prescribed medication for OA. " Patients were asked "Which of the following options best describes your overall satisfaction with the prescribed medicine(s) for your osteoarthritis?" (options: very satisfied, 1; somewhat satisfied, 2; neither satisfied nor dissatisfied, 3; somewhat dissatisfied, 4; very dissatisfied, 5). Sample size: $n=572{ }^{\mathrm{b}}$ Patients were asked

physician ratings of expectations of effectiveness with $54.6 \%$ of 557 ratings matched (Cohen's weighted kappa $=0.48 ; p<0.0001)$.

\section{DISCUSSION}

This study found that, for patients, the presence of comorbid other musculoskeletal or painful conditions was among the factors associated with greater overall satisfaction with currently prescribed medication for $\mathrm{OA}$, and the most troublesome joint being other than a knee, hip, or back was among the factors associated with greater expectation of effectiveness; exercise was positively associated with both these patient-reported outcomes. For their physicians,
"How is your current medicine(s) meeting your level of expectation in relation to how effective it is for your OA?" (options: it is a great deal more effective than I expected, 1; it is more effective than I expected, 2; it matches my expectations, 3; it is less effective than I expected, 4; it is much less effective than I expected, 5). Sample size: $n=557 . O A$ osteoarthritis

the presence of comorbid other musculoskeletal or painful conditions was among the factors associated with greater overall satisfaction; the most troublesome joint being a knee was associated with both greater satisfaction and higher rating for the medication meeting the patient's expectations of efficacy, and adverse events/tolerability issues were negatively associated with both these outcomes. For both patients and their physicians, satisfaction with prescribed medication for OA was most strongly associated with efficacy.

The association between greater pain and dissatisfaction with medications supports previous findings $[5,17,18]$. A study of patients with knee OA receiving a range of oral, topical, and intra-articular medications found that 


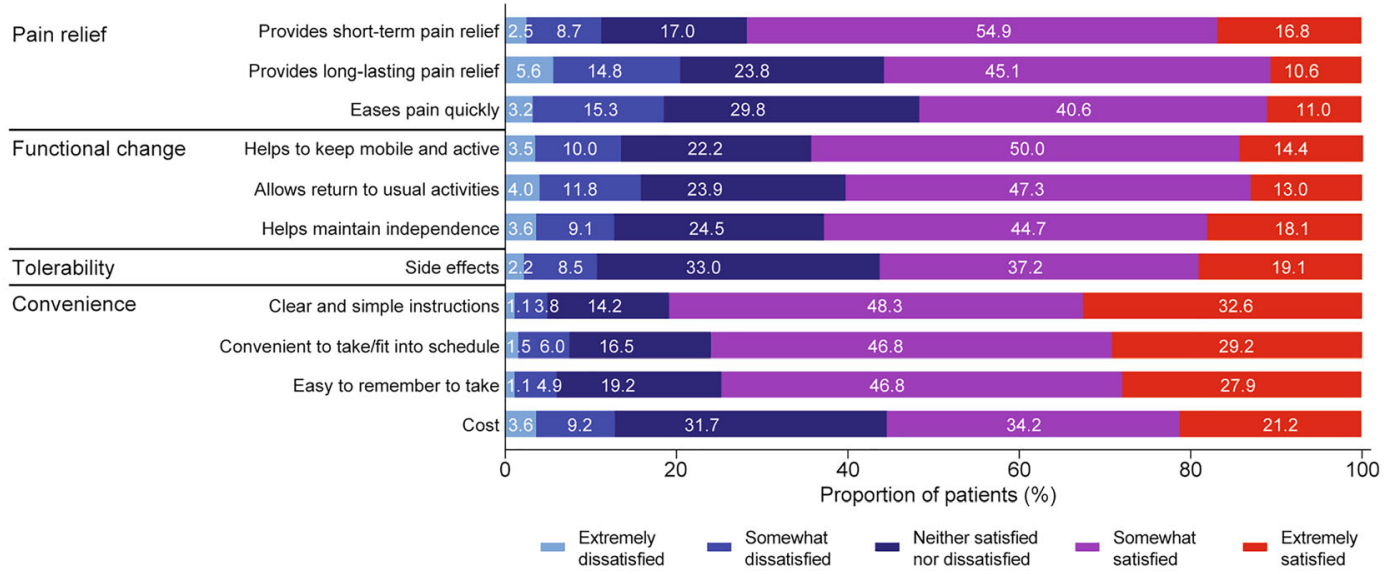

Fig. 2 Patient-reported satisfaction with attributes of currently prescribed medication for OA. Patients rated their satisfaction with different attributes of medications from 1 to 5 (extremely dissatisfied, 1; somewhat dissatisfied, 2; neither dissatisfied nor satisfied, 3; somewhat satisfied, 4; extremely satisfied, 5), in response to "How satisfied are you with your prescribed medicine(s) in relation to...": provides short-term pain relief (sample size for this response, $n=552$ ), provides long lasting pain relief $(n=554)$, eases your pain quickly $(n=554)$, helps keep you mobile and active $(n=550)$, allows you to return to your usual activities $(n=552)$, helps maintain your independence $(n=552)$, the side effects of the medicine $(n=551)$, has clear and simple instructions $(n=549)$, is convenient to take in terms of fitting into your schedule $(n=551)$, is easy to remember to take $(n=551)$, the cost of my medicine $(n=552)$. OA osteoarthritis

satisfaction with medications for their OA pain. The reasons for this are unclear, as this group was heterogenous and sample sizes for individual conditions were small.

Better health status was strongly associated with greater patient-reported satisfaction in the models of pain relief and functional change. Issues of adverse events/tolerability were associated with patient-reported dissatisfaction in the model of satisfaction with medication tolerability. When considering patient-reported satisfaction with the convenience of medications for OA, taking multiple medications for $\mathrm{OA}$ was associated with dissatisfaction and taking multiple medications for concomitant conditions was associated with satisfaction. The roles of ethnicity and age in patients' satisfaction with the convenience of medications for OA may warrant further investigation.

Few demographic factors were found to be associated with overall satisfaction in the current study. Female patients were associated with greater, and unemployment was associated with worse, physician-rated (but not patient-reported) overall satisfaction. A study of shoulder conditions"), both patients and physicians in 

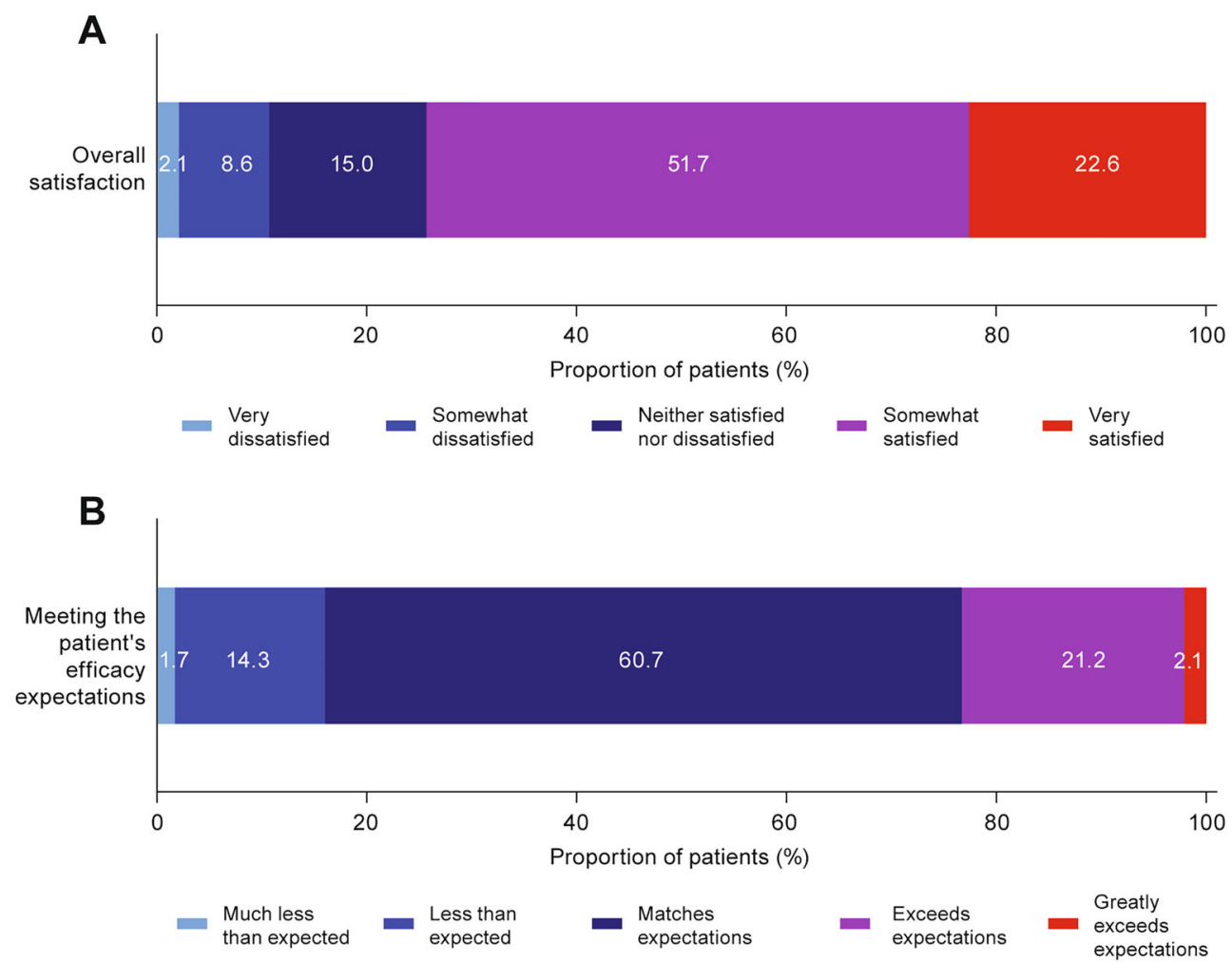

Fig. 3 Physician-rated a overall satisfaction with currently prescribed medication for $\mathrm{OA}^{\mathrm{a}}$ and $\mathbf{b}$ views of how the currently prescribed medication for $\mathrm{OA}$ is meeting the patient's expectation of efficacy ${ }^{\mathrm{b}}$. ${ }^{\mathrm{a}}$ Physicians were asked "Which of the following statements best describes your satisfaction with the patient's prescribed therapy (to treat their OA pain)?” (options: very satisfied, 1; somewhat satisfied, 2; neither satisfied nor dissatisfied, 3; somewhat

pain found that pain in female patients may be underestimated by others [29]; it is feasible that pain relief may be overestimated in female patients too, consequently increasing physician-rated satisfaction with treatment. In a previous study of patient-reported treatment satisfaction, being employed predicted higher global satisfaction scores, and being female predicted higher effectiveness satisfaction scores [5]. Exercise was identified as important for patient-reported (but not physician-rated) overall satisfaction in the current study. The benefit of exercise for patients with $\mathrm{OA}$ is clear [4], and by enhancing self-efficacy for managing pain $[30,31]$ exercise could feasibly influence satisfaction with pharmaceutical treatments. dissatisfied, 4; very dissatisfied, 5). Sample size: physician ratings for 572 patients. ${ }^{\text {b }}$ Physicians were asked "How do you believe the current drug therapy regimen meets the patient's own expectations in relation to efficacy achieved?" (options: greatly exceeds expectations, 1; exceeds expectations, 2; matches expectations, 3; less than expected, 4; much less than expected, 5). Sample size: physician ratings for 572 patients. $O A$ osteoarthritis

The current analyses were an assessment of overall medication with many patients receiving more than one class of prescribed OA medication (Supplementary Material, Table S3). This may explain why bivariate analyses (satisfied vs neutral/dissatisfied) were not significant for most classes of OA medication. Generally, classes of medication were not associated with satisfaction: although opioids and corticosteroids were identified as important covariates by LASSO (Supplementary Material, Fig. S2), significance in one final model was only achieved for opioids (i.e., with the attribute of functional change) (Table 4).

Patients and their physicians agreed on many of the factors that were associated with 
worse overall satisfaction (including lack of efficacy, pain, and OA that was deteriorating) and better overall satisfaction (including comorbid other musculoskeletal or painful conditions, and physician-reported best control achieved), although the relative importance sometimes differed. Differences were identified where the association was significant only for patients or only for physicians. For patients (but not physicians), exercise was associated with better overall satisfaction and drug interactions/comorbidities were associated with worse overall satisfaction. For physicians (but not patients), adverse events/tolerability issues, treatment-resistant pain, unemployment not due to OA, and two lines of OA medication were associated with worse overall satisfaction, whereas female patients and obesity were associated with greater overall satisfaction. The most troublesome joint being a knee was significantly associated with physician-rated overall satisfaction; in contrast, joint location did not reach significance in the model of patientreported overall satisfaction. Physicians and policy makers may consider these findings to optimize medication-related consultations to achieve better patient satisfaction.

There are few published data on factors influencing expectations of effectiveness of medications for OA. Generally, the factors that were important for level of expectation were similar to those that were important for level of overall satisfaction. Notably, exercise (for patients) and the most troublesome joint being a knee and adverse events/tolerability issues (for physicians) were significant in both expectations and satisfaction models. Patient-reported worse expectation of effectiveness was associated with the use of OTC medication. A previous study using the same database found that $40.1 \%$ of patients were currently recommended by their physician to take OTC medications, and OTC acetaminophen use was more frequent in those with more severe pain [32]. It is feasible that the minimal short-term benefit of acetaminophen for OA [33], or inadequate dosages of other OTC medications, may have influenced patient-reported expectation of effectiveness of OA medications in the current analyses. Lack of efficacy was most strongly associated with worse expectation of effectiveness in the current study, from the perspective of both patient rating of effectiveness and physicians' rating of how the medication had met the patient's efficacy expectations.

The factors for inclusion in the multivariate models were identified by LASSO linear regression, a methodology that supports simple models. LASSO is particularly beneficial where there is a paucity of prior evidence, in that the method enables testing of large numbers of potentially correlated factors and the identification of the most important ones. This allows for a more manageable set of factors for formulating further hypotheses testing. The magnitude of the absolute standardized betas provided by the current analyses are not informative with respect to clinical meaningfulness, but do reflect the relative strength of the factors within each model (a factor with a standardized beta of 0.2 has twice the importance of a factor with a beta of 0.1).

The current study had some limitations. The data reflect the wording of the survey questions, which may be interpreted differently (including the criteria each patient uses to define effectiveness, and each physician's definition of efficacy and best [pain] control). The analyses may over-represent satisfied patients, because physician-rated overall satisfaction was lower for the 103 patients excluded because of missing patient-rated overall satisfaction responses than for the included patients. Assessing satisfaction with currently prescribed medication for OA required that all patients were currently prescribed medication: patients who were not taking medication (e.g., because they chose not to, or their symptoms did not warrant it) or who were dissatisfied enough with treatment to stop taking prescription medication were not included in the current analyses. The Adelphi OA DSP consists of patients seen by a small sample of US primary care physicians, rheumatologists, and orthopedic surgeons willing to participate in research and may not be generalizable to the whole OA population, other countries, or all specialties caring for patients with OA. The methods used in this study were for hypothesis generation rather than hypothesis testing; therefore, a causal relationship between factors 
and outcomes cannot be inferred. The factors included in the models were obtained during a cross-sectional assessment, but some pertained to the current time and others were historical, being based on physician chart review or patient recall. Covariates considered for inclusion in the models were based on bivariate analysis for one outcome (patient-reported overall satisfaction with medications for $\mathrm{OA}$ ) and therefore some key factors associated with the other outcomes of interest (e.g., expectation of effectiveness) may not have been included in those analyses.

\section{CONCLUSION}

Although efficacy was strongly associated with both patients' and physicians' satisfaction with medication, other factors were also important. Among the factors associated with greater overall satisfaction with, and expectation of effectiveness of, medications for OA, exercise was important for patients and tolerability and the most troublesome joint being a knee were important for physicians. Patients and their physicians generally agree on many of the factors associated with satisfaction with prescribed medication for $\mathrm{OA}$, but the relative importance sometimes differed and exceptions were identified. Understanding the factors that are associated with patient satisfaction, and how these differ from those associated with physician satisfaction, could aid shared decision-making, optimize treatment, and improve satisfaction for patients with OA. Further study of the differences between patient and physician perspectives of treatment satisfaction could be informative for quality care initiatives.

\section{ACKNOWLEDGEMENTS}

Funding. This study was funded by Pfizer and Eli Lilly and Company. The study was conducted by Adelphi Real World. Publication fees (expenses to the journal for Rapid Service and Open Access) were funded by Pfizer and Eli Lilly and Company. Pfizer Inc and Eli Lilly and
Company contributed to the study design. In their role as authors, employees of Pfizer and Eli Lilly were involved in the interpretation of data, preparation, review, and approval of the manuscript and the decision to submit for publication, along with their co-authors. The study sponsors approved the manuscript from an intellectual property perspective but had no right to veto the publication.

Authorship. All named authors meet the International Committee of Medical Journal Editors (ICMJE) criteria for authorship for this article, take responsibility for the integrity of the work as a whole, and have given their approval for this version to be published.

Author Contributions. All authors contributed to the conception or design of the study/analysis plan. Authors from Adelphi conducted the study including the acquisition and analysis of data. All authors contributed to the interpretation of data. All authors contributed to drafting the manuscript and revising it critically for important intellectual content. All authors approved the final version to be published and agree to be accountable for all aspects of the work in ensuring that questions related to the accuracy or integrity of any part of the work are appropriately investigated and resolved.

Medical Writing, Editorial, and Other Assistance. Medical writing support was provided by Kim Russell, PhD, of Engage Scientific Solutions (Horsham, UK) and was funded by Pfizer and Eli Lilly and Company.

Prior Presentation. Some of these data were presented at Osteoarthritis Research Society International World Congress, April 29-May 1, 2021.

Disclosures. Thomas J Schnitzer reports clinical research study support (Pfizer, Lilly, Regeneron, Galapagos, Taiwan Liposome Corporation, Anika Therapeutics) and fees for consultancy/advisory boards (Pfizer, Lilly, GSK, AstraZeneca, Galapagos, Merck). Rebecca L Robinson is an employee and stockholder of Eli 
Lilly and Company. Sophie Barlow and Mia Berry are employees of Adelphi Real World, which received funding from Pfizer and Eli Lilly and Company to conduct the study. Andrew Bushmakin, Joseph C Cappelleri, and Leslie Tive are employees of Pfizer with stock and/or stock options. During the study, Jessica Jackson was an employee of Adelphi Real World, which received funding from Pfizer and Eli Lilly and Company to conduct the study. James Jackson is an employee of Adelphi Real World, which received funding from Pfizer and Eli Lilly and Company to conduct the study. Lars Viktrup is an employee and stockholder of Eli Lilly and Company.

Compliance with Ethics Guidelines. The Adelphi DSP methodology was granted exemption from requiring ethics approval by the Western Institutional Review Board as it was considered to pose minimal risk to patients and physicians. All patients provided consent.

Data Availability. The data that support the findings of this study are available from Adelphi Real World, but restrictions apply to the availability of these data, which were used under license for the current study and so are not publicly available. However, data are available from the authors upon reasonable request and with permission from Adelphi Real World.

Open Access. This article is licensed under a Creative Commons Attribution-NonCommercial 4.0 International License, which permits any non-commercial use, sharing, adaptation, distribution and reproduction in any medium or format, as long as you give appropriate credit to the original author(s) and the source, provide a link to the Creative Commons licence, and indicate if changes were made. The images or other third party material in this article are included in the article's Creative Commons licence, unless indicated otherwise in a credit line to the material. If material is not included in the article's Creative Commons licence and your intended use is not permitted by statutory regulation or exceeds the permitted use, you will need to obtain permission directly from the copyright holder. To view a copy of this licence, visit http:// creativecommons.org/licenses/by-nc/4.0/.

\section{REFERENCES}

1. Paskins Z, Sanders T, Hassell AB. Comparison of patient experiences of the osteoarthritis consultation with GP attitudes and beliefs to OA: a narrative review. BMC Fam Pract. 2014;15:46.

2. Geenen R, Overman CL, Christensen R, et al. EULAR recommendations for the health professional's approach to pain management in inflammatory arthritis and osteoarthritis. Ann Rheum Dis. 2018;77:797-807.

3. Bannuru RR, Osani MC, Vaysbrot EE, et al. OARSI guidelines for the non-surgical management of knee, hip, and polyarticular osteoarthritis. Osteoarthr Cartil. 2019;27:1578-89.

4. Kolasinski SL, Neogi T, Hochberg MC, et al. 2019 American College of Rheumatology/Arthritis Foundation Guideline for the management of osteoarthritis of the hand, hip, and knee. Arthritis Care Res (Hoboken). 2020;72:149-62.

5. Ueda K, Sasaki N, Goren A, et al. Treatment satisfaction with pharmaceutical interventions in Japanese adults with osteoarthritis and chronic knee pain: an analysis of a web-based survey. Clin Interv Aging. 2018;13:2179-91.

6. Van Zaanen Y, Hoorntje A, Koenraadt KLM, et al. Non-surgical treatment before hip and knee arthroplasty remains underutilized with low satisfaction regarding performance of work, sports, and leisure activities. Acta Orthop. 2020;91:717-23.

7. Taylor SD, Everett SV, Taylor TN, Watson DJ, Taylor-Stokes G. A measure of treatment response: patient and physician satisfaction with traditional NSAIDs for osteoarthritis control. Open Access Rheumatol. 2013;5:69-76.

8. Carlson J, Youngblood R, Dalton JA, Blau W, Lindley C. Is patient satisfaction a legitimate outcome of pain management? J Pain Symptom Manage. $2003 ; 25: 264-75$.

9. Shikiar R, Rentz AM. Satisfaction with medication: an overview of conceptual, methodologic, and regulatory issues. Value Health. 2004;7:204-15.

10. Wong WS, Chow YF, Chen PP, Wong S, Fielding R. A longitudinal analysis on pain treatment satisfaction among Chinese patients with chronic pain: predictors and association with medical adherence, 
disability, and quality of life. Qual Life Res. 2015;24:2087-97.

11. Hirsh AT, Atchison JW, Berger JJ, et al. Patient satisfaction with treatment for chronic pain: predictors and relationship to compliance. Clin J Pain. 2005;21:302-10.

12. Conaghan PG, Serpell M, McSkimming P, Junor R, Dickerson S. Satisfaction, adherence and health-related quality of life with transdermal buprenorphine compared with oral opioid medications in the usual care of osteoarthritis pain. Patient. 2016;9:359-71.

13. Timmerman L, Stronks DL, Huygen FJ. The relation between patients' beliefs about pain medication, medication adherence, and treatment outcome in chronic pain patients: a prospective study. Clin J Pain. 2019;35:941-7.

14. Kravitz RL. Patients' expectations for medical care: an expanded formulation based on review of the literature. Med Care Res Rev. 1996;53:3-27.

15. Lenderking WR. Brief reflections on treatment satisfaction. Value Health. 2005;8(Suppl 1):S2-5.

16. Gunaratne R, Pratt DN, Banda J, Fick DP, Khan RJK, Robertson BW. Patient dissatisfaction following total knee arthroplasty: a systematic review of the literature. J Arthroplasty. 2017;32:3854-60.

17. Gimenez S, Armada B, Iturralde Iriso J, Ginel Mendoza L, Fernández-Morales B. Clinical management of patients with hip and knee osteoarthritis: patient satisfaction with treatment switch. Rheumatol Int. 2014;34:823-32.

18. Dworkin RH, Jensen MP, Gould E, et al. Treatment satisfaction in osteoarthritis and chronic low back pain: the role of pain, physical and emotional functioning, sleep, and adverse events. J Pain. 2011;12:416-24.

19. Anderson P, Benford M, Harris N, Karavali M, Piercy J. Real-world physician and patient behaviour across countries: disease-specific programmes-a means to understand. Curr Med Res Opin. 2008;24: 3063-72.

20. Theiler R, Spielberger J, Bischoff HA, Bellamy N, Huber J, Kroesen S. Clinical evaluation of the WOMAC 3.0 OA Index in numeric rating scale format using a computerized touch screen version. Osteoarthr Cartil. 2002;10:479-81.

21. Herdman M, Gudex C, Lloyd A, et al. Development and preliminary testing of the new five-level version of EQ-5D (EQ-5D-5L). Qual Life Res. $2011 ; 20: 1727-36$.

22. Rosner B. Fundamentals of biostatistics. 8th ed. Boston, MA: Cengage Learning; 2015.

23. Mehta CR, Patel NR. A network algorithm for performing Fisher's exact test in $\mathrm{r} \times \mathrm{c}$ contingency tables. J Am Stat Assoc. 1983;78:427-34.

24. Bendel RB, Afifi AA. Comparison of stopping rules in forward "stepwise" regression. J Am Stat Assoc. 1977;72:46-53.

25. Mickey RM, Greenland S. The impact of confounder selection criteria on effect estimation. Am J Epidemiol. 1989;129:125-37.

26. Tibshirani R. Regression shrinkage and selection via the Lasso. J R Stat Soc Ser B Stat Methodol. 1996;58: 267-88.

27. Schroeder LD, Sjoquist DL, Stephan PE. Understanding regression analysis: an introductory guide. Thousand Oaks, CA: SAGE; 2017.

28. Fleiss JL, Cohen J. The equivalence of weighted kappa and the intraclass correlation coefficient as measures of reliability. Educ Psychol Meas. 1973;33: 613-9.

29. Zhang L, Losin EAR, Ashar YK, Koban L, Wager TD. Gender biases in estimation of others' pain. J Pain. 2021;22:1048-59.

30. Somers TJ, Wren AA, Shelby RA. The context of pain in arthritis: self-efficacy for managing pain and other symptoms. Curr Pain Headache Rep. 2012;16: 502-8.

31. Mihalko SL, Cox P, Beavers DP, et al. Effect of intensive diet and exercise on self-efficacy in overweight and obese adults with knee osteoarthritis: the IDEA randomized clinical trial. Transl Behav Med. 2019;9:227-35.

32. Nalamachu SR, Robinson RL, Viktrup L, et al. Multimodal treatment patterns for osteoarthritis and their relationship to patient-reported pain severity: a cross-sectional survey in the United States. J Pain Res. 2020;13:3415-25.

33. Machado GC, Maher CG, Ferreira PH, et al. Efficacy and safety of paracetamol for spinal pain and osteoarthritis: systematic review and meta-analysis of randomised placebo controlled trials. BMJ. 2015;350:h1225. 